\title{
MODELAGEM E SIMULAÇÃO DE UM REATOR EM LEITO FIXO PARA PRODUÇÃO DE BIODIESEL VIA CATÁLISE HETEROGÊNEA A PARTIR DO ÓLEO DE DENDÊ
}

\author{
C. B. Sousa, V. A. M. Gomes, S. M. P. Lucena \\ Universidade Federal do Ceará, Departamento de Engenharia Química \\ E-mail para contato: caiobragasousa@gmail.com
}

\begin{abstract}
RESUMO - Um reator em leito fixo em escala industrial para produção contínua biodiesel com carga $8000 \mathrm{~kg} / \mathrm{h}$ de óleo de dendê e metanol, usando a rota de transesterificação heterogênea e dados cinéticos disponíveis, foi modelado e simulado com o uso de software numérico. $\mathrm{O}$ modelo matemático do reator foi desenvolvido segundo as hipóteses de regime permanente, velocidade intersticial e porosidade constantes, força motriz linear para a transferência de massa, e operação isotérmica sem dispersão axial. Três casos de estudos foram simulados com a variação dos seguintes parâmetros: temperatura, velocidade espacial horária de líquido (LHSV) e razão álcool/ óleo. Para cada caso, os perfis de conversão do óleo ao longo do reator foram construídos. Deste perfis, foi possível concluir que as melhores de condições de operação foram a seguinte: temperatura de $338 \mathrm{~K}$, LHSV igual 0,25 e razão molar álcool/óleo igual a 13,7. Tais condições poderão ser utilizadas para obtenção de dados técnicos-econômicos de uma unidade industrial completa.
\end{abstract}

\section{INTRODUÇÃO}

Por motivos econômicos, ambientais e geopolíticos, diversos países têm concentrado suas atenções em termos de pesquisa científica no intuito de alterarem suas matrizes energéticas, que em nível global é fortemente dependente dos combustíveis fósseis: petróleo, gás natural e carvão mineral. Tal atenção tem-se voltado principalmente para as fontes renováveis de energia, que diferentemente dos combustíveis fósseis, não estão atreladas a prazos de esgotamento. O biodiesel, produzido a partir da transesterificação de óleos vegetais ou gorduras animais com um álcool de cadeia curta, em geral metanol ou etanol, apresenta-se como uma destas fontes (XIAO et al., 2012).

O processo convencional de produção de biodiesel, responsável pela maior parte da produção atualmente, é a transesterificação de óleos vegetais refinados usando metanol como álcool de cadeia curta e um catalisador alcalino homogêneo, principalmente o $\mathrm{NaOH}$ (Di Serio et al., 2008). Tal processo é conhecido como rota homogênea básica, ou simplesmente rota homogênea.

Embora a transesterificação pela rota homogênea básica conduza a altas conversões do óleo vegetal em mono-alquil ésteres, esta apresenta uma séria de desvantagens: é energointensiva; o catalisador tem que ser removido do efluente do reator, o que implica o uso de processos de purificação complexos, com a produção de consideráveis volumes de água 
residual; ácidos graxos livres presentes óleo interferem no processo, em virtude da ocorrência de reações competitivas de saponificação (Atadashi et al., 2011).

Outro problema comum referente a transesterificação homogênea está na utilização de óleo vegetal refinado, sendo que o custo da matéria prima graxa representa cerca $75 \%$ dos custos totais de produção (Morais, 2010). No cenário brasileiro, a maioria das plantas operam com óleo de soja, o que causa um déficit econômico, sendo este é suprido somente por subsídios governamentais. No entanto, a diversificação das matérias primas pode ser uma saída para o impasse, aja visto que algumas fontes de matéria prima, como o óleo de dendê, apresentam grandes disponibilidades de óleo. A palmeira de dendê pode chegar a níveis de produção de até 6 toneladas/hectare. No entanto, o principal problema referente ao óleo de dendê refere-se a sua acidez, que em geral é elevada, fato que pode inviabilizar o uso deste óleo pela rota homogênea (Santana, 2008).

Neste contexto, a transesterificação usando catalisadores heterogêneos, ou rota heterogênea, apresenta vantagens sobre a rota homogênea. A mais importante delas refere-se a exclusão de seguidas etapas de purificação, que envolvem a neutralização do meio reacional, lavagem e secagem do biodiesel e destilação, que na catálise homogênea são etapas fundamentais. Como consequência, há uma redução no número de equipamentos de processo, redução na quantidade de água requerida, redução na quantidade de efluentes com custos ambientais reduzidos, e corrosão em equipamentos (XIAO et al., 2012). Outra importante vantagem da rota heterogênea diz respeito a sua insensibilidade em relação a acidez do óleo, o que torna esta rota ideal para a transesterificação do óleo de dendê em biodiesel. Além disso o processo também produz uma glicerina com elevada pureza, com pelo menos $98 \% \mathrm{~m} / \mathrm{m}$, sendo que esta possui um maior valor agregado (Di Serio et al., 2008).

Atualmente existem poucos trabalhos publicados com a modelagem e a simulação de processos de produção de biodiesel com catálise heterogênea. Existe na verdade uma profusão de trabalhos científicos propondo novos catalisadores heterogêneos para o processo, mas destes trabalhos, poucos apresentam dados cinéticos para os catalisadores, sendo estes fundamentais para o projeto de reatores (Fogler, 2012).

Portanto, a proposta deste trabalhos a de modelar e simular um reator em leito fixo industrial para produção de biodiesel, com carga de óleo de dendê e metanol usando a rota de transesterificação heterogênea e dados cinéticos e de constantes de equilíbrio do modelo de Eley-Rideal disponíveis no trabalho de Xiao et al., (2010). Os parâmetros do leito foram obtidos do trabalho de Xiao et al., (2012).

\section{MODELAGEM DO REATOR}

O modelo matemático para o reator foi desenvolvido com as hipóteses simplificadoras de regime permanente, velocidade intersticial e porosidade constantes, força motriz linear para a transferência de massa, e operação isotérmica sem dispersão axial (Cavalcante Jr., 1998). Uma seção transversal do reator de comprimento $d z$ e área $A$ constantes foi tomada como volume de controle como está esquematizado na Figura 1. 


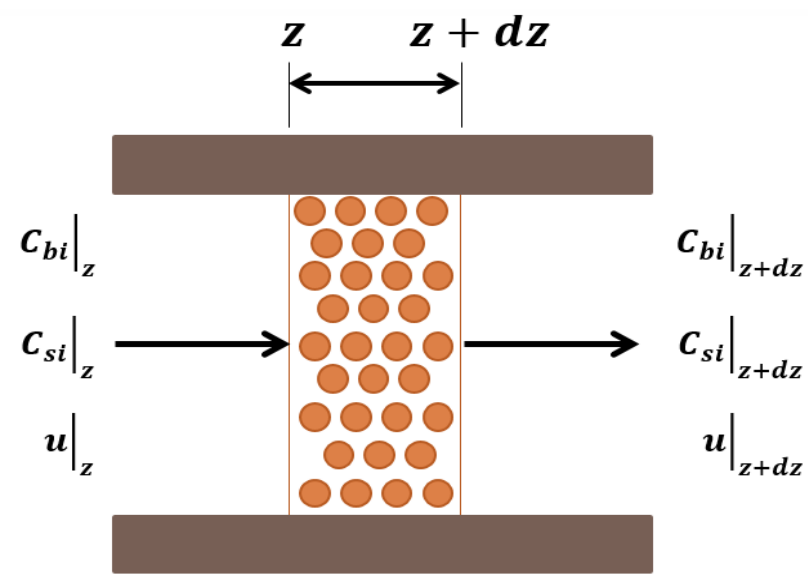

Figura 1 - Seção transversal do reator com comprimento $d z$.

Balanços molares nas fases fluida e sólida foram realizados para desenvolver a seguinte equação do modelo:

$$
-(L H S V \times L) \frac{d C_{b i}}{d z}=v_{i} \rho_{b} \eta r_{e}
$$

Onde $L H S V$ é a Velocidade Espacial Horária de Líquido, $C_{b i}$ a concentração do i-ésimo componente na fase líquida, $v_{i}$ o coeficiente estequiométrico do i-ésimo componente, $\rho_{b}$ a massa específica aparente do sólido do leito, $\eta$ o fator de efetividade interno para a transferência de massa na partícula de catalisador, e $r_{e}$ a velocidade de reação incluindo os efeitos externos de transferência de massa (Fogler, 2012).

O modelo de Eley-Rideal foi utilizado para representar a cinética da reação, sendo descrito pela seguinte equação:

$$
r_{e}=\eta_{e} \frac{k\left(C_{A} C_{B}-\frac{1}{K} \frac{C_{C}^{3} C_{D}}{C_{B}^{2}}\right)}{1+K_{B} C_{B}+K_{C} C_{C}}
$$

Onde $C_{A}, C_{B}, C_{C}$ e $C_{D}$ são as concentrações molares de óleo, metanol, glicerol e biodiesel respectivamente em kmol. $\mathrm{m}^{-3}$ na fase líquida, $k$ a constante de velocidade em $\mathrm{m}^{6} . \mathrm{kmol}^{-1} . \mathrm{kg}$ cat. $\mathrm{s}^{-1}, K$ a constante de equilíbrio, e $K_{B}$ e $K_{C}$ as constantes de equilíbrio de adsorção no catalisador para o metanol e o glicerol em $\mathrm{m}^{3} \cdot \mathrm{kmol}^{-1}$, respectivamente, e $\eta_{e} \mathrm{o}$ fator de efetividade externo para a transferência de massa no filme externo.

O fator de efetividade externo $\eta_{e}$ foi definido a partir da seguinte relação entre a velocidade de reação $r_{e}$ e a velocidade de reação desprezando os efeitos da transferência de massa $r$ : 
$\eta_{e}=\frac{r_{e}}{r}$

E o fator de efetividade externo $\eta$ foi definido a partir da seguinte relação entre a velocidade de reação $r_{e}$ e a velocidade de reação considerando os efeitos da transferência de massa interna e externa $r_{e i}$ :

$$
\eta=\frac{r_{e i}}{r_{e}}
$$

A seguinte equação foi utilizada para obter os valores de $\eta$ como função do Módulo de Thiele $\Phi$ :

$$
\eta=\frac{1}{\Phi}\left[\frac{1}{\tanh (3 \Phi)}-\frac{1}{3 \Phi}\right]
$$

E $\Phi$ foi calculado pelas seguintes equações

$$
\begin{aligned}
& \Phi=\frac{V_{p}}{S_{p}} \sqrt{\frac{r_{e}}{C_{A 0} D_{e f}}} \\
& \frac{D_{i f} \mu_{f}}{T}=\frac{7,4 \times 10^{-8}\left(\varphi_{s} M_{s}\right)^{1 / 2}}{V_{i}^{0,6}} \\
& D_{e f}=\frac{D_{i f}}{\delta} \theta
\end{aligned}
$$

Onde $V_{p}$ e $S_{p}$ são o volume e a área superficial de partícula em $m^{3}$ e $m^{2}$, respectivamente, $C_{A 0}$ a concentração molar de óleo na entrada do reator $(z=0), D_{i f}$ a difusividade do i-ésimo componente na fase fluida em $\mathrm{cm}^{2} / \mathrm{s}, \mu_{f}$ a viscosidade da fase fluida em $c P, \varphi_{s}$ o fator de associação do solvente igual 1,9 no caso o metanol, $M_{s}$ a massa molar do solvente, $V_{i}$ o volume molar do soluto na temperatura ade ebulição em $\mathrm{cm}^{3} / \mathrm{mol}$, no caso o óleo, $D_{e f}$ a difusividade efetiva do i-ésimo componente nos poros do catalisador, $\delta$ a tortuosidade e $\theta$ a porosidade na partícula do catalisador. As Equações 2 a 8 foram definidas de acordo com o trabalho de Xiao et al., (2012). 


\section{PARÂMETROS DO MODELO}

Alguns parâmetros das Equações 1 a 8 obtidos dos trabalhos de Xiao et al., (2010) e Xiao et al., (2012). Entretanto, outros parâmetros foram definidos de acordo com uma condição de escala industrial, pois nos trabalhos supracitados, os parâmetros estiveram relacionados a uma condição de escala laboratorial. A Tabela 1 mostra os valores dos parâmetros usados neste trabalho.

Tabela 1 - Parâmetros usados na simulações do reator

\begin{tabular}{|c|c|}
\hline Dados de Difusividade & Valores \\
\hline$D_{A f}\left(m^{2} / s\right):$ difusividade do óleo & $2,26 \times 10^{-10}$ \\
\hline$D_{B f}\left(m^{2} / s\right):$ difusividade do metanol & $3,00 \times 10^{-10}$ \\
\hline$D_{C f}\left(m^{2} / s\right):$ difusividade do glicerol & $3,33 \times 10^{-10}$ \\
\hline$D_{D f}\left(m^{2} / s\right):$ difusividade do biodiesel & $2,12 \times 10^{-10}$ \\
\hline \multicolumn{2}{|l|}{ Dados Cinéticos e de Constantes de Equilíbrio } \\
\hline 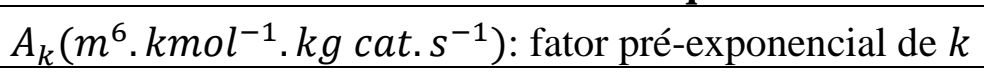 & $8,69 \times 10^{15}$ \\
\hline$E_{k}(J / m o l):$ energia de ativação de $k$ & 112923 \\
\hline$A_{B}\left(m^{3} \cdot \mathrm{kmol}^{-1}\right):$ fator pré-exponencial de $K_{B}$ & $7,63 \times 10^{-07}$ \\
\hline$E_{B}(J / m o l):$ energia de ativação de $K_{B}$ & $-37536,6$ \\
\hline$A_{C}\left(m^{3} \cdot \mathrm{kmol}^{-1}\right):$ fator pré-exponencial de $K_{C}$ & $3,27 \times 10^{-10}$ \\
\hline$E_{C}(J / m o l):$ energia de ativação de $K_{C}$ & $-61238,9$ \\
\hline \multicolumn{2}{|l|}{ Parâmetro do Leito Reacional } \\
\hline$D(m)$ : diâmetro do reator & 0,30 \\
\hline$L(m)$ : comprimento do reator & 40 \\
\hline$\rho_{p}(\mathrm{~kg} / \mathrm{m} 3):$ massa específica das partículas & 2306,4 \\
\hline$d_{p}(m)$ : diâmetro das partículas de catalisador & 0,005 \\
\hline$\varepsilon:$ porosidade do leito & 0,52 \\
\hline$\delta:$ tortuosidade & 4,1 \\
\hline$\theta:$ porosidade da partícula & 0,0713 \\
\hline
\end{tabular}

A viscosidade $\mu_{f}$ da fase fluida foi calculada a partir do método UNIFAC-VISCO descrito em Poling et al., (2004).

\section{RESULTADOS E DISCUSSÕES}

As equações do modelo foram resolvidas computacionalmente pelo de método de Runge-Kutta de $4^{\circ}$ ordem. Foram realizados estudos de caso com a variação dos parâmetros $L H S V$, razão molar álcool/óleo $m$ e temperatura isotérmica de reação $T$. A variação destes parâmetros seguiu o mesmo padrão apresentado no trabalho Xiao et al., (2012), de tal forma a validar e comparar os resultados destes trabalho com os do autor. As Figura 1 a 3 mostram os 
resultados das simulações, onde os perfis de conversão do óleo ao longo do reator foram construídos para cada caso.

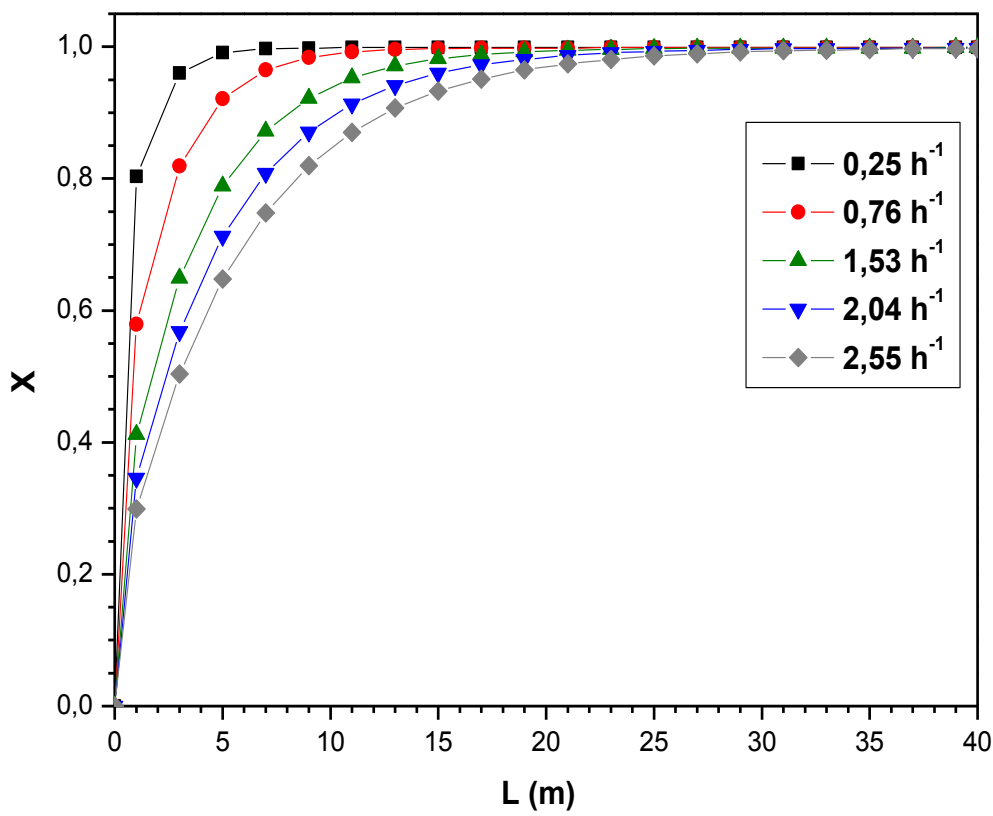

Figura 1 - Variação do LHSV.T $=343 \mathrm{~K}$ e $m=22,73$.

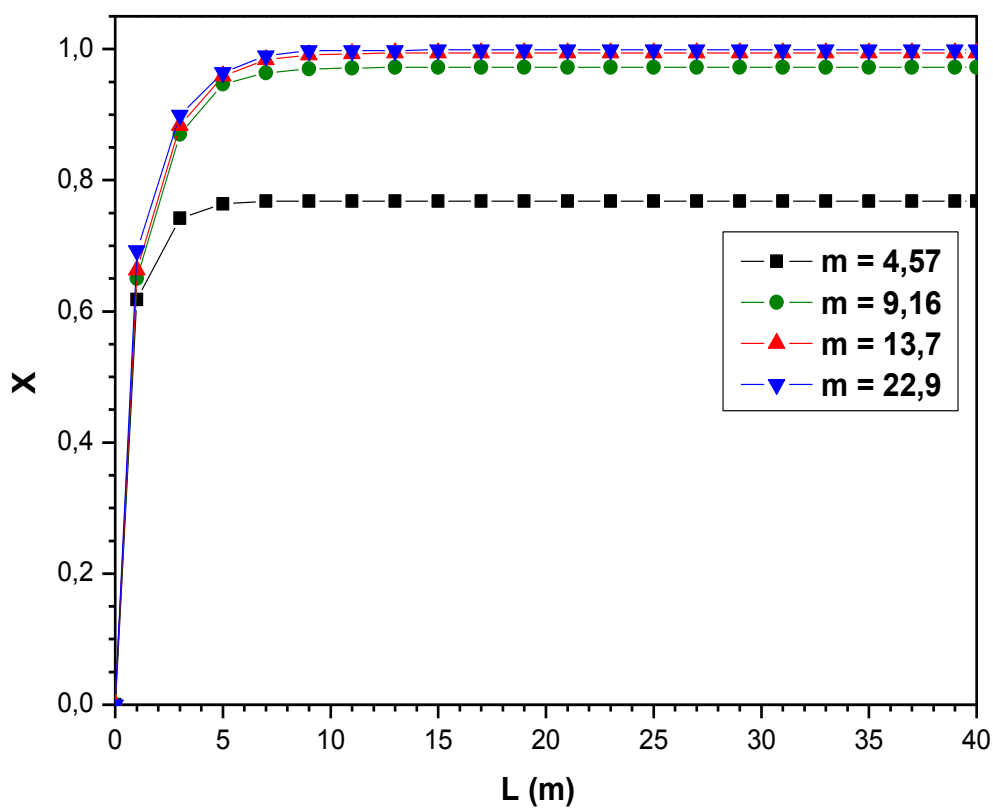

Figura 2 - Variação da razão álcool/óleo $m . L H S V=0,25 \mathrm{~h}^{-1}$ e $T=338 \mathrm{~K}$. 


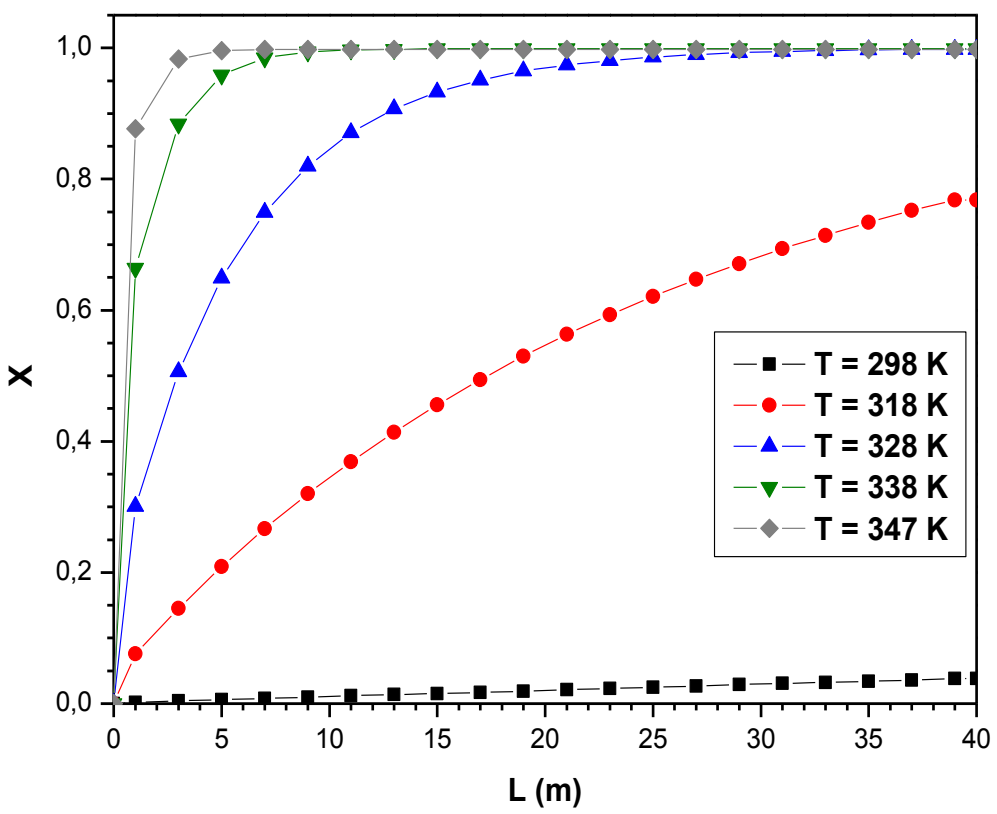

Figura 3 - Variação da temperatura $T . L H S V=0,25 \mathrm{~h}^{-1}$ e $m=22,73$.

A Figura 1 demonstra que o decréscimo do $L H S V$ aumenta o valores de conversão ao longo do reator. Este comportamento se explica pelo de que o LHSV é o inverso do tempo espacial tomando como referência a alimentação a $60^{\circ} \mathrm{F}$ (Fogler, 2012). Como o tempo espacial é igual ao tempo de residência médio das moléculas no reator, quanto maior o seu valor, ou inversamente, quanto menor os valor de $L H S V$, maiores serão os valores de conversão ao longo do reator, como mostrado pelo perfis.

Já na Figura 2, sabe-se a reação de transesterificação tende para um equilíbrio termodinâmico (XIAO et al., 2010). Estequiometricamente, a reação requer uma razão molar álcool/óleo $m=3$. Entretanto, tal razão estequiométrica não é adequada para se conseguir conversões altas em relação ao óleo no reator, de tal forma que é necessária a utilização de uma estratégia para deslocar o equilíbrio no sentido dos produtos. A estratégia mais adequada do ponto de vista econômico é utilizar um excesso em álcool para deslocar o equilíbrio (WEST et al., 2008). Logo, quanto maior razão molar álcool/óleo, maiores serão as conversões ao longo do reator.

$\mathrm{Na}$ Figura 3, o aumento de temperatura favoreceu o aumento dos valores dos parâmetros cinéticos e da constante de equilíbrio, esta última relacionada ao deslocamento do equilíbrio termodinâmico no sentido dos produtos.

Por último, os perfis de conversão também mostraram comportamentos similares aos mostrados no trabalho de Xiao et al., (2012). Este fato serviu para validar o modelo proposto. 


\section{CONCLUSÕES}

Baseado nos resultados apresentados neste trabalho, foi possível concluir o seguinte:

- O modelo foi satisfatório em prever o comportamento da conversão do óleo ao longo do reator;

- O resultados demonstram a validade do modelo proposto;

- Da análise do perfis, foi possível concluir que as melhores condições operacionais foram: temperatura igual $338 \mathrm{~K}, L H S V$ igual a 0,25 e razão molar álcool/óleo igual a 13,7.

\section{REFERENCIAS}

ATADASHI, I. M.; AROUA, M. K.; AZIZ, A. A. Biodiesel separation and purification: A review. Renewable Energy, v. 36, p. 437-443, 2011.

CAVAlCANTE Jr., C.L. Separação de Misturas por Adsorção: dos Fundamentos ao Processamento em Escala Comercial. Fortaleza: Tese (Prof. Titular), Universidade Federal do Ceará, Departamento de Engenharia Química, 1998.

DI SERIO. M. et al. Heterogeneous Catalysts for Biodiesel Production. Energy \& Fuels, v. 22, p. 207-217, 2008.

FOGLER, H. S. Elementos de Engenharia das Reações Químicas. Rio de Janeiro: LTC Editora, 2009.

MORAIS, S. et al. Simulation and life cycle assessment of process design alternatives for biodiesel production from waste vegetable oils. Cleaner Production, v. 18, p. 1251$1259,2010$.

SANTANA, G. C. S. Simulação e Análise de Custos na Produção de Biodiesel a Partir de Óleos Vegetais. Campinas, SP: Unicamp, 2008. Originalmente apresentada como tese de doutorado.

WEST, A. H.; POSARAC, D.; ELLIS, N. Assessment of four biodiesel production processes using HYSYS.Plant. Bioresource Technology, v. 99, p. 6587-6601, 2008.

XIAO, Y. et al. Kinetics of the Transesterification Reaction Catalyzed by Solid Base. Energy \& Fuels, v. 24, p. 5829-5833, 2010.

XIAO, Y. et al. Experimental and Modeling Study of Continuous Catalytic Transesterification to Biodiesel in a Bench-Scale Fixed-Bed Reactor. Industrial \& Engineering Chemistry Research, v. 51, p. 11860-11865, 2012. 